\title{
Tricuspid regurgitation following central line insertion in a patient undergoing haemodialysis
}

\author{
John T Walsh, Peter Glennon, Peter M Schofield
}

A 38-year-old woman with insulin-dependent diabetes and chronic renal failure on long-term peritoneal dialysis was admitted following blockage of her dialysis catheter. A right internal jugular line (Quinton catheter) was inserted for haemodialysis while awaiting reinsertion of a peritoneal catheter. Two weeks later she developed a fever with signs of new tricuspid regurgitation. Transoesophageal echocardiography revealed a right atrial mass (figure).

\section{Department of Cardiology, Papworth Hospital, Papworth Everard, Cambridgeshire CB3 8RE, UK J T Walsh \\ P Glennon \\ P M Schofield}

Accepted 23 April 1998

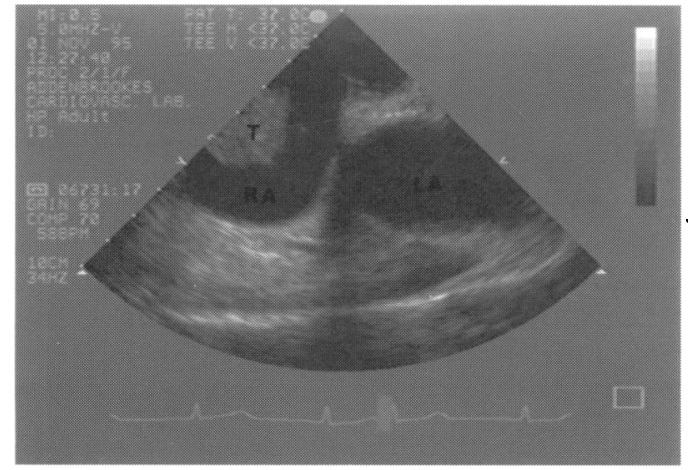

\section{Questions}

1 What is the clinical diagnosis?

2 Name the most probable infecting organism.

Figure Transoesophageal echocardiogram. $T=$ thrombus $R A=$ right atrium $L A=$ left atrium 
Answers

QUESTION 1

Central line infection with infected right atrial thrombus and tricuspid valve endocarditis.

QUESTION 2

Staphylococcus aureus is the most probable infecting organism.

\section{Follow-up}

The patient was started on flucloxacillin and was transferred for cardiac surgery. The atrial clot was removed and a vegetation on the tricuspid valve leaflet resected. She remained on intravenous antibiotics for 6 weeks postoperatively and made a slow recovery, having required hospitalisation for nearly 8 months.

\section{Discussion}

Infection is common in patients with chronic renal failure and the second leading cause of death. ${ }^{1}$ Infective endocarditis is an uncommon, yet often lethal, complication of access site infection in patients with renal failure. ${ }^{23}$ This patient was one of five with chronic renal failure referred to a regional cardiothoracic centre over an 8-month period, three of whom died as a direct consequence of vascular access site infection; the remaining two required cardiac surgery.

The primary source of infection in patients with chronic renal failure often relates to the vascular access site and can affect almost any organ. ${ }^{24}$ In all the five patients referred to above, a temporary haemodialysis catheter had been inserted via a central vein and was the most likely source of infection. Dialysis catheter procedures in the referring renal unit have since been reviewed. The importance of strict asepsis during insertion and manipulation of catheters has been re-emphasised; patients deemed likely to require haemodialysis have atrio-venous fistulae fashioned in advance wherever possible and if central venous access is unavoidable then the duration of this is kept to a minimum with periodic changes of site. In the 18 months since these cases were highlighted and stricter asepsis policies enforced

1 Lowrie EG, Lazarus JM, Mocelin AJ, et al. Survival of patients undergoing chronic haemodialysis and renal transpatients undergoing chronic haemodialysis

2 Cross AS, Sleibegel RT. Infective endocarditis and access site infections in patients on haemodialysis. Medicine 1976;55:453-66.

\section{Learning points}

- bacterial endocarditis occurs in up to $6.6 \%$ of patients with chronic renal failure undergoing haemodialysis

- vascular access sites, in particular central venous catheters, are the commonest source of infection

- Staphylococcus aureus is the most probable infecting organism in this group of patients

- strict aseptic procedures during and after central line insertion are important factors in the reduction of risk

there have been no further cases of dialysiscatheter-associated endocarditis.

Although prophylactic antibiotics have been suggested as a means of reducing the frequency of access site infection there are no controlled data to support such a policy. Indeed the widespread use of antibiotic therapy in such circumstances may increase mortality by inducing antibiotic-resistant strains. Similarly, there are no data to support the use of local antibiotic therapy to the vascular access site; eradication of nasal staphylococcal carriage in patients on chronic haemodialysis may, however, be useful in some cases.

As the number of patients requiring dialysis continues to rise, the incidence of central line infection seems likely to increase. Renal units are well aware of the risk of bacterial endocarditis in this group of patients but general physicians need also to consider the serious consequences of haemodialysis-associated endocarditis when central venous access is required. Awareness of such an outcome will be a factor in reduction of risk.

\section{Final diagnosis}

Central line infection with Staphylococcal aureus causing right atrial thrombus and tricuspid valve endocarditis.

Keywords: Staphylococcal aureus infection; catheterassociated endocarditis; haemodialysis

We thank Dr John Bradley, Consultant Renal Physician, for allowing us to prepare this report. Dr Glennon is supported by the British Heart Foundation.

3 Nsouli KA, Lazarus M, Schoenbaum SC, Gottlieb $M N$, Lowrie EG, Schocair M. Bacteraemic infection in haemodialysis. Arch Intern Med 1979;139:1255-8.

4 Kaslow RA, Zellner SR. Infection in patients on maintenance haemodialysis. Lancet 1972;117. 